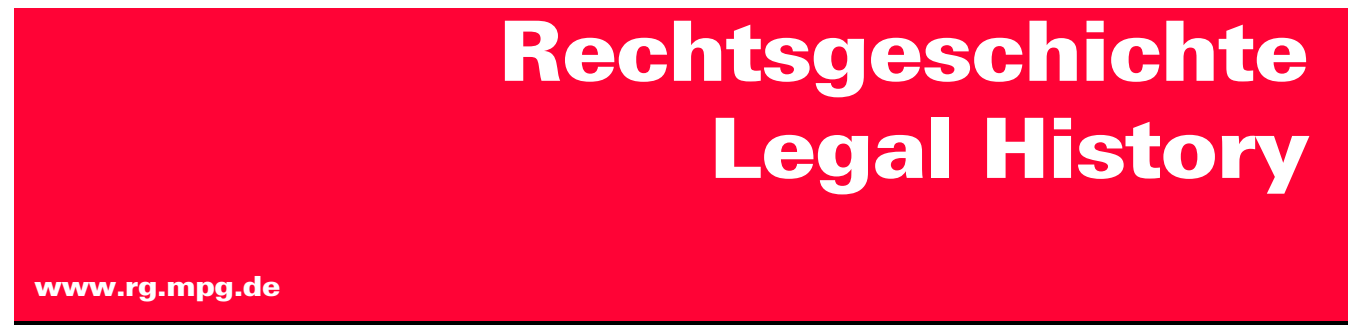

http://www.rg-rechtsgeschichte.de/rg27

Zitiervorschlag: Rechtsgeschichte - Legal History Rg 27 (2019)

$\operatorname{Rg} 272019$

$297-300$

http://dx.doi.org/10.12946/rg27/297-300

\title{
Karla Escobar*
}

\section{Agresivamente histórico y global}

[Aggressively Global and Historical]

* Max Planck Institute for European Legal History, Frankfurt am Main / Universidad de los Andes, klescobarh@uniandes.edu.co 
some than his use of an prefatory captatio benevolentiae in favor of an "argumentative and discursive transhistoricity« might suggest. The same holds true for the ex-ante and applicative aiming toward a "post-honor society«, which at best deprives the reader the delight of drawing one's own conclusions. The recourse to contemporary socalled honor killings as an uncivilized phenomenon remains overly simplistic, especially since it is the postulated legal character of such acts of revenge that could be problematized and seems to be mutating into a parallel system of justice. On the other hand, Ruch's examples from classical antiquity repeatedly demonstrate how well suited they are to research of anachronistic structures. For instance, the interstate conflict between Athens and Mytilene clearly shows that even (or particularly) democratic communities require narratives of legitimation that go beyond the mere process of decision-making when it comes to the use of violence - a situation encountered with greater frequency in recent times.

The study offers neither fundamentally new paradigms to legal history, in the sense of Westbrook's formulation of them as a desideratum, nor policy recommendations for modern »dis-honorable" societies. Moreover, the author, unfortunately, does not provide an index to help make the impressive number of sources and examples more accessible. Ruch's history of emotion and law is nevertheless worth all the effort because it enhances our view of the eternally "new « sources of antiquity, and thus of ourselves. If emotions prove to be bound not only by culture and time, but also by law, and if we accept that law is a process of communication and knowledge, then the syllogism still offers us some leeway.

\section{Karla Escobar \\ Agresivamente histórico y global*}

Global Histories and Cultures of Statehood, libro editado por John L. Brooke, Julia Strauss y Greg Anderson, es una propuesta interesante de reflexión interdisciplinaria sobre las formaciones estatales en un marco temporal amplísimo: desde la Antigüedad hasta el siglo XX. Los capítulos que componen el libro fueron presentados en un programa de dos años en la Universidad Estatal de Ohio. La publicación es claramente el producto de un ejercicio de discusión colectiva de una red académica con sede en los Estados Unidos y, por tal motivo, la mayoría de sus miembros pertenecen a instituciones académicas en este país.

Las diferentes trayectorias de sus editores Brooke, desde la historia intelectual y ambiental;
Strauss, desde la Ciencia Política y Anderson, desde la teoría crítica - se corresponden con las variadas trayectorias académicas de los contribuyentes. Considero que el aporte más importante de este libro es precisamente su interés por presentar diferentes aproximaciones - incluso aquellas contrarias entre sí -, en las que los académicos han interpretado históricamente los procesos de formación estatal a partir de marcos cronológicos y geográficos diversos. Tal variedad de aproximaciones es articulada en la introducción y conclusión, en donde los editores cumplen con la difícil tarea de hacer un balance sobre los principales puntos de encuentro y desencuentro entre trabajos tan diversos.

* John Brooke et al. (eds.), State

Formations. Global Histories and

Cultures of Statehood, Cambridge:

Cambridge University Press 2018,

xxiii + 383 p., ISBN 978-1-108-40394-8 
No resulta fácil, entonces, reseñar un trabajo de esta naturaleza, en la medida en que los aportes abren distintos hilos de discusión y plantean debates y retos metodológicos diversos, muchas veces inconmensurables entre sí. Debido a ello, en esta reseña haré una lectura crítica no de cada uno de los artículos, sino de la obra en su conjunto, en relación con los propósitos de la misma, planteados por los editores en su Prefacio: »This volume offers an overview of approaches to the state for the new century. We are aggressively global and historical. Where typically such projects are confined to the kinds of states that are >modern ‘, , Western « or both, we consider here the full range of state experience over five millennia, and in every major region of the world« (xvii).

Para cumplir con esta noble tarea, los editores estructuran el libro en cuatro secciones. La primera está dedicada a lo que llaman las »definiciones«. En este aparte se le ofrece al lector algunas aproximaciones teóricas sobre el estado. La segunda sección se titula "fundaciones« y se centra en analizar las diferentes experiencias de fundación de los estados en marcos temporales y geográficos diversos. La tercera, que titulan como "agendas", busca responder a las estrategias mediante las cuales los estados, institucionalmente, han dado respuesta a las metas impuestas por ellos mismos, así como a las demandas de sus súbditos/ciudadanos; la última parte está dedicada a lo que los autores catalogan como "membresía«, sección en la que buscan dar cuenta de las múltiples maneras en las que los sujetos lograron participar en los procesos de formación estatal y las sutiles tensiones y efectos que esta participación conllevó.

La sección »definiciones« se encuentra compuesta por tres artículos, todos ellos centrados en Europa. El primero, de Quentin Skinner, centra su reflexión en el análisis de la teoría política anglófona, partiendo del trabajo de Hobbes. Seguidamente está el trabajo de Bob Jessop, quien, desde la tradición gramsciana, propone comprender el poder estatal a partir de entender el estado en tanto relación social. La sección se cierra con el trabajo de Greg Anderson, quien hace énfasis en la naturaleza del estado como un fenómeno eminentemente moderno. Si bien este último trabajo busca establecer un diálogo con formaciones estatales de China y Grecia, tales reflexiones están dadas para comprender la experiencia »occidental«, aunque sin definir muy bien qué se entiende por ello. Los tres trabajos son novedosos y sus aportes son retomados, en diferentes niveles, por los autores de los capítulos siguientes.

La sección »Fundaciones" está compuesta por seis capítulos, la mayoría de ellos estudios de caso y, a diferencia de la primera sección, goza de una mayor variedad geográfica, aunque no cronológica. Dos enfocan su análisis en el estudio de las formaciones estatales en la Antigüedad; los otros se centran en el periodo moderno. Los trabajos se distribuyen de forma relativamente equitativa (mas no representativa) en los diferentes continentes: dos para Asia (India y Valle del Indo, que abarca las actuales Afganistán, Paquistán y Nordeste Indio), uno para África (Costa Swahili en el Sudeste Africano), uno para Europa (Italia), uno para América (Estados Unidos) y cierra con un trabajo comparativo entre Rusia, Irán y Alemania.

El aparte dedicado a las »Agendas« está compuesto también por seis trabajos: uno dedicado al Imperio Romano, tres a los Estados Unidos de América, uno a China y otro a Inglaterra; la mayoría de ellos están centrados en el siglo XIX. En contraposición, y quizás para balancear la perspectiva estadounidense, en la sección de »membresías" se dedica un artículo a Mesopotamia, otro a China, otro a Gran Bretaña y Burma, otro a Kurdiztán, otro a Colombia y uno a la India.

Con base en esta breve descripción de la estructura del texto, quisiera volver a revisar el citado párrafo introductorio comenzando por su primera frase: "This volume offers an overview of approaches to the state for the new century.« Efectivamente esta obra sirve para darnos una visión general sobre diferentes aproximaciones sobre el estado. Se compromete con la difícil tarea de poner en diálogo no solamente distintas disciplinas, sino también marcos teóricos y cronológicos diversos. Los textos introductorios proponen pautas de análisis interesantes y son útiles para ayudarnos a pensar fenómenos contextuales concretos. Tal es el caso, por ejemplo, de la idea de "state effect" (Anderson), así como la idea del estado como relación social (Jessop), las cuales están en diálogo con las recientes discusiones que han cuestionado el estudio del estado desde la mera formalidad institucional, para entender los procesos de formación estatal en la historia. En este sentido, si bien el libro aporta una buena »mirada general«, que puede ser útil en el aula, resulta pretencioso pensar que tal generalidad pueda guiar nuestras disquisiciones en el »nuevo siglo«. Quizás sería más aterrizado decir que busca sintetizar muchas discusio- 
nes que se produjeron en la historiografía estadounidense de las últimas décadas y, aun así, la síntesis no es exhaustiva.

»We are aggressively global and historical«: Es claro que la preocupación por lo global es el corazón de este libro y que, debido a ello, hubo un ejercicio consciente de tratar de incluir académicos que trabajaran diferentes territorialidades con la idea de "poner sobre la mesa « las diferentes lecturas sobre fenómenos que han sido catalogados bajo la categoría de »formación estatal « y, desde allí, ver como las discusiones teóricas se trasforman en los ámbitos contextuales. Lo "global«, en este caso, no busca entenderse en su versión relacional, en tanto fenómeno que se produce y resignifica de diferente manera sobre territorios distintos, sino es una estrategia narrativa que permite construir un escenario de posibilidades analíticas plurales y, en ese sentido, tal apelación a la globalidad es una apuesta interesante. Sin embargo, no deja de molestar la desproporcionalidad a partir de la cual ese escenario de posibilidades analíticas es construido. $\mathrm{Al}$ parecer, para los editores la inclusión de sólo un trabajo para todo el continente africano y de un sólo trabajo para la región latinoamericana no levanta un mínimo ápice de inconformidad. Y no estoy hablando de la necesidad de haber incluido trabajos sobre el periodo pre-colombino (como creen los editores que es su punto débil), sino la total falta de inclusión de la profusa historiografía que se ha producido, en las últimas décadas, sobre los procesos modernos de construcción estatal en estos territorios. Esta ausencia debilita profundamente las posibilidades analíticas que el trabajo dice querer construir. La historiografía sobre los procesos de formación estatal en América Latina, para dar sólo un ejemplo, ha venido cuestionando las ideas de »modernidad « y »occidentalidad « que parecen preocupar tanto a los autores y que, sin necesidad de abarcar una cronología y marco geográfico tan amplio (o quizás debido a ello), han logrado hacer lecturas más sofisticadas.

Lo anterior se relaciona con la idea de ser "agresivamente históricos«. Incluir un marco cronológico de cinco milenios no es necesariamente ser más histórico; de hecho, lo que se puede evidenciar en el texto es que el análisis histórico, como tal, se debilita profundamente al deambular a través de periodos tan distantes unos de otros. De allí que, usualmente los capítulos dedicados al Mundo Antiguo parezcan más como un objeto decorativo en función de la conjurada »agresividad histórica" pero que poco son incluidos en los análisis de la introducción y conclusión a partir de sus propias inquietudes y preguntas disciplinares. Así, lo »agresivamente global« se corresponde más bien con lo »limitadamente histórico«, no porque los trabajos no sean históricos, que lo son, sino porque no logran establecer entre ellos diálogos que permitan complejizar la mirada local, tan propia del estudio de caso.

Estas particularidades del trabajo nos llevan a la crítica de la tercera premisa: »Where typically such projects are confined to the kinds of states that are smodern', ,Western ‘ or both, we consider here the full range of state experience over five millennia, and in every major region of the world «. Creo que aquí está el punto más débil del texto, precisamente por todo lo anterior. El escrito, si bien especifica en sus conclusiones que uno de sus grandes hallazgos es confirmar que la emergencia del estado moderno no es una historia solamente europea (hallazgo poco novedoso, por cierto), parte de la idea preconcebida de que es allí donde se origina, se extiende y se resignifica de manera más o menos exitosa, en los diferentes territorios. De allí que la sección de »definiciones « tenga como eje a Europa. Así, aunque se especifique que el estado moderno no es una historia exclusivamente europea, no se pone en duda que su marco teórico deba serlo. La distribución del libro y de las territorialidades que aborda da cuenta de ello. Así, no hay una reflexión mínima sobre lo que significó ser moderno en diferentes territorialidades - que en varias ocasiones no coincidió con los procesos que se estaban dando en Europa - como lo han mostrado algunos trabajos históricos recientes para el caso latinoamericano. La obra, al seguir trabajando sobre la idea de una Europa que »llevó « el estado moderno a otros rincones del planeta en donde adquirió diferentes características, obnubila el proceso relacional mediante el cual las dinámicas económicas, sociales, culturales y políticas - en escalas locales, regionales y globales diversas -, terminaron por definir qué es lo que era un estado moderno, qué significaba ser moderno, quienes eran sus abanderados y los desarrollos que esas dinámicas produjeron dentro de los propios procesos de formación estatal, tanto para los estados europeos - en su diversidad - como para el resto del mundo.

A pesar de estas críticas, considero que este libro es muy valioso para los académicos que estudiamos formaciones estatales en diferentes periodos y desde varias disciplinas. Para los historiadores, 
es una herramienta útil para salir fácilmente de la zona de confort y deambular a través de los tiempos, sin la necesidad de construir falsas linealidades. A través de la lectura de los artículos individuales, es fácil encontrarnos con preguntas sugerentes, con nuevos y viejos conceptos, así como con otras formas de interpretación. Para los historiadores del derecho, por su parte, la lectura de esta obra brinda una oportunidad para ver los múltiples lugares en los que el derecho reside y los métodos que distintas disciplinas tienen para hacerle hablar y comprenderlo. Esta obra es, al fin y al cabo, un ejercicio de diálogo que debe continuarse, sobre todo en estos tiempos de hiper-especialización de los saberes, y que, además, espero se siga nutriendo, ojalá también agresivamente, de nuevos aportes y perspectivas.

\section{Georg May}

\section{Kanonistik im Spiegel von Kanonisten*}

Der Herausgeber will einen Überblick über die Kirchenrechtswissenschaft durch die Schilderung von Leben und Werk ihrer gelehrten Betreiber geben. Die ersten 100 Seiten stellen Personen vor, die keine Kanonisten waren. Man kann schwerlich von Rechtsgelehrten sprechen vor Entstehung der Rechtswissenschaft. So finden sich bei Paulus zwar Texte, die kanonistisch bedeutsam sind, aber ein Kanonist war er nicht. Augustinus war Praktiker des Kirchenrechts, dessen Texte nicht selten als autoritative Stellungnahmen betrachtet wurden (wobei der ihm gewidmete Beitrag ausgiebig zu Papst Franziskus abschweift). Justinian war Gesetzgeber. Isidor von Sevilla war ein emsiger Sammler, aus dessen Werken reiche Belehrung zu schöpfen ist, vor allem für Kirche und Klerus, aber die kanonistische Bearbeitung nahm er selbst nicht vor. Hinkmar von Reims war im Recht seiner Zeit zu Hause, wie vor allem sein Werk über den Ehestreit Lothars II. zeigt. Er war in der Lage, die Synoden, an denen er beteiligt war, zu prägen. Aber systematisch arbeitender Kirchenrechtler war auch er nicht. Die von der pseudoisidorischen Fälschergruppe produzierten Texte brachten keinen wissenschaftlichen Fortschritt. Bei der Darstellung Abaelards überwuchert die Lebensgeschichte das Werk. Mit Gratian betreten wir sicheren Bo- den. Der instruktive Beitrag von Matthias Pulte über ihn gibt den Fragen über seine Person erheblich mehr Raum als der Darstellung seines Werkes. Stephan von Tournai leitete die notwendige Unterscheidung von Theologie und Kanonistik ein (126-130). Neu in einer Sammlung von Kanonisten ist die Aufnahme des Hugo von Sankt-Viktor in der Darstellung durch Rainer Berndt (131-141). Wohltuend berührt die gerechte Würdigung von Bonifaz VIII. durch Stefan Ihli (142-153). Von Johannes Teutonicus, bearbeitet von Thomas Zotz (160-165), hätte man gern mehr erfahren. Daniela Müller ist um sachliche Darstellung des Bernard Gui bemüht (166-176). Was Martin Luther in einer Sammlung von Kanonistenporträts zu suchen hat (182-192), ist nicht ohne Weiteres einsichtig. Er hat bekanntlich das kirchliche Recht in Frage gestellt. Der Autor, Heinz-Meinolf Stamm OFM, bestreitet seinen Beitrag hauptsächlich aus langen Zitaten anderer Gelehrter. Robert Bellarmin (203-214) ist doch wohl vorwiegend als Kontroverstheologe zu würdigen, daneben als Vertreter des ius publicum ecclesiasticum.

Mit Ferdinand Walter ist der Herausgeber im 19. Jahrhundert angekommen. Die stete Lernbereitschaft dieses Bonner Kanonisten wird zutreffend gewürdigt (215-219). Ansgar Hense entreißt

* Philipp Thull (Hg.), 60 Porträts aus dem Kirchenrecht. Leben und Werk bedeutender Kanonisten, Sankt Ottilien: EOS-Verlag 2017, 664 S., ISBN 978-3-8306-7824-3 